\title{
Analgesic Medicine Utilization in Older People in New Zealand from 2005 to 2013
}

\author{
Prasad S. Nishtala ${ }^{1} \cdot$ Sekbong $\mathrm{Oh}^{1} \cdot$ Daniel Kim ${ }^{1} \cdot$ Natalie Chun $^{1} \cdot$ \\ Siti Fatimah binti Kamis ${ }^{1} \cdot$ Kuan-Cia Kiu $^{1}$
}

Published online: 8 May 2015

(c) The Author(s) 2015. This article is published with open access at Springerlink.com

\begin{abstract}
Background Older people (aged 65 years and above) consume more analgesics compared with other age groups. Because of the aging of the New Zealand population, it is important to ascertain the trends of analgesic use at a population-level.

Objective The aim of this population-based study was to describe and characterize the utilization of analgesic medicines in older people in New Zealand from 2005 to 2013.

Methods Repeated cross-sectional analysis of populationlevel dispensing data was conducted from 2005 to 2013. Dispensing data were obtained from Pharmaceutical Claims Data Mart (Pharms), Ministry of Health, extracted using encrypted national health index numbers as unique identifiers and categorized by the World Health Organization Collaborating Centre for Drug Statistics Methodology (WHOCC) Anatomical Therapeutic Chemical (ATC) classification system. Utilization of analgesics was measured in defined daily dose (DDD) per 1000 older people per day (TOPD).

Results Overall, analgesic medicine utilization increased by $5.44 \%$ from 2005 to 2013 . Analgesic utilization increased from 233.66 to 246.36 DDD/TOPD. The increased utilization was mainly driven by analgesics and antipyretics. An increased utilization of analgesics was seen in the $85+$ age group. Females consumed more analgesics compared with males. Differences in analgesic utilization across the District Health Boards (DHBs) persisted over the
\end{abstract}

Prasad S. Nishtala

prasad.nishtala@otago.ac.nz

1 School of Pharmacy, University of Otago, PO Box 56, Dunedin 9054, New Zealand 9-year period. Utilization of anti-migraines was stable over the 9-year period.

Conclusions This population-level utilization study showed a $5.44 \%$ increase in utilization of analgesic medicines in older people in New Zealand from 2005 to 2013. Important findings were that (1) females utilized more analgesics compared with males; (2) non-steroidal anti-inflammatory drug (NSAID) utilization decreased over the years; and (3) the highest utilization was reported in the $85+$ group. Further research is warranted to examine the drivers influencing analgesic use in New Zealand.

\section{Key Points}

Overall, analgesic utilization in people aged 65 and above increased by $5.44 \%$ between 2005 and 2013 .

Analgesic utilization in females was higher than in males.

Non-steroidal anti-inflammatory drug (NSAID) utilization decreased between 2005 and 2013.

\section{Introduction}

Analgesics have been a mainstay in the treatment of acute and chronic pain $[1,2]$. The use of analgesics is of particular importance in older people aged 65 years and above, as this group of people have a higher incidence of both acute and chronic pain compared with younger age groups, affecting as much as $76 \%$ of community-dwelling 
older people [3]. Older people take analgesics for persistent pain brought about by musculoskeletal [3, 4], cephalgia or gastrointestinal disorders [4]. Over the past few decades, there has been an increase in the utilization of analgesics, especially opioid analgesics, among people in developed countries $[1,5,6]$. One study reported that two out of three older adults aged more than 75 years took at least one regular analgesic medicine [7]. In a population-level study conducted in Spain, the opioid utilization increased 14-fold from 1992 to 2006 [1]. In Israel, the utilization of morphine, oxycodone, pethidine, methadone, and fentanyl increased by $47 \%$ in 2008 when compared with 2000 [8]. Oxycodone utilization alone was $50 \%$ higher in 2008 than in $2000[2,8]$.

Several factors could influence variations in opioid analgesic utilization across countries. In addition to sociodemographic factors, regulatory and ethical guidelines are fundamental factors that influence access to opioid analgesics. In New Zealand, weak opioids such as codeine are available over-the-counter (OTC) when in combination with other active ingredients. Codeine as a single ingredient and all other opioid analgesics are classified as prescription-only controlled drugs, where strict regulations are enforced [9]. Subsidy and funding also play significant roles in prescribing these medicines. The Pharmaceutical Management Agency (PHARMAC) is the New Zealand government agency that decides which medicines are subsidized [10]. PHARMAC manages budget, funding and access restrictions for medicines [11]. Hence, analgesic medicine utilization profiles in New Zealand would be quite dependent upon decisions made by PHARMAC.

Older people are prone to experiencing adverse effects associated with analgesics compared with their younger counterparts [12]. Opioid analgesic use in older people may be associated with an increased risk of falls due to their adverse effects on the central nervous system, which include dizziness, sedation and/or syncope [12, 13]. Polypharmacy is common in older people [12, 14], and the concomitant use of analgesics can contribute to clinically significant drug-drug interactions and adverse reactions [14]. Use of non-steroidal anti-inflammatory drugs (NSAIDs) in older people has been associated with an increased prevalence of gastrointestinal bleeding and incidence of peptic ulcer, as well as an increased risk of cardiovascular events. The increased risk of adverse drug events in older people is due to pharmacokinetic changes brought about by aging, reduced renal clearance and hepatic metabolism, as well as pharmacodynamic changes leading to increased sensitivity to certain analgesics [12].

Studies have shown that age, sex, socioeconomic status and ethnicity influence the frequency of pain symptoms and consequently utilization of analgesics $[15,16]$. Hence, it is important to ascertain the trends of analgesic use among these groups. To date, there has been very limited research on analgesic utilization among older people in New Zealand by age, sex, ethnicity, geographical locations and socioeconomic status.

\section{Objective}

The main objective of this study was to describe and characterize the national trend in utilization of analgesic medicines in New Zealand's older populations from 2005 to 2013 , stratified by year, age, sex, ethnicity and District Health Board (DHB), using the World Health Organization Collaborating Centre for Drug Statistics Methodology (WHOCC) Anatomical Therapeutic Chemical (ATC) classification system.

\section{Methods}

This study was approved by the Human Ethics Committee of the University of Otago, New Zealand (approval number HD14/15).

\subsection{Study Design}

A repeated cross-sectional analysis of population-level dispensing data was conducted from 1 January 2005 to 31 December 2013 for individuals aged 65 years and above. Data were extracted on analgesic medicines used (WHOCC/ATC code N02 analgesics: N02A opioids, N02B other analgesics and antipyretics, N02C anti-migraine preparations; and M01 anti-inflammatory and antirheumatic products: M01A NSAIDs), using encrypted national health index numbers as the unique identifier for each individual, and were analysed for utilization with regards to year, age, sex, ethnicity and DHBs. The dispensing data included all funded analgesics used in this age group.

\subsection{Data Source}

De-identified dispensing claims data for all individuals 65 years and over for the period 2005-2013 were obtained from Pharmaceutical Claims Data Mart (Pharms), New Zealand Ministry of Health. Analgesic medicines were categorized using the ATC classification system of the WHOCC and Norwegian institute of public health. The ATC classification system has been sub-grouped into five levels with anatomical, therapeutic, pharmacological, chemical and chemical substance subgroups, in descending order. The ATC therapeutic classes analysed at broad levels were opioids (N02A), other analgesics and 
antipyretics (N02B), anti-migraine preparations (N02C) and NSAIDs (M01A).

\subsection{Statistical Analysis}

Defined daily dose (DDD), a weighted technical utilization measure, was used to describe the number of older people on a defined standard daily dose (formulation not factored) per 1000 older people per day (TOPD). These standard doses are set by WHOCC for each medicine, updated every 3 years and used only for the purpose of examining medicine utilization or use and to compare utilization with other countries. The methodology for analysing combination products was adapted from the "Guidelines for ATC classification and DDD assignment" published by the WHO Collaborating Centre for Drug Statistics Methodology. In this study, DDDs for combinations were the DDDs for the individual analgesic ingredients. For example, for the combination product of paracetamol and codeine, the DDD for codeine was computed and included in the analyses for total analgesic consumption. Utilization measures and use rates by year, age, sex, ethnicity and DHB were measured as DDD per TOPD. DDDs were computed for each analgesic medicine consumed by an individual, yearly, per annual population size of New Zealand from 2005 to 2013. Data on the older resident population in New Zealand for each year in the time period 2005-2013 were obtained from Statistics New Zealand [17]. Statistical analysis was completed using IBM SPSS Statistics software version 22 .

\section{Results}

\subsection{Overall Analgesic Medicine Utilization Over Time}

The analysis showed that the national utilization of analgesic medicines in 2005 was $233.66 \mathrm{DDD} / \mathrm{TOPD}$, which increased to 246.36 in 2013. Overall, analgesic utilization increased by $5.44 \%$, with an average DDD/TOPD of 242.85 over the 9 -year period. Analgesic medicine utilization peaked in 2012, as shown in Fig. 1.

\subsection{Utilization of Analgesic Medicines by Age, Sex, Ethnicity and DHB}

Analgesic utilization by sex, age (5-year bands), ethnicity and DHB showed increasing trends over the 9-year period, and higher utilization was observed in 2013 than in 2005. The highest DDD/TOPD was observed in those aged 85 years and above, as shown in Fig. 2, with an average of

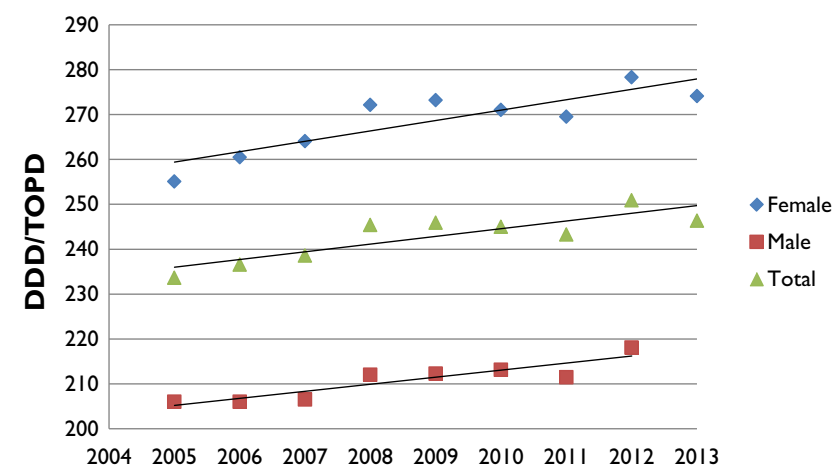

Fig. 1 DDD/TOPD utilization based on sex from 2005 to 2013. $D D D$ defined daily dose, TOPD 1000 older people per day

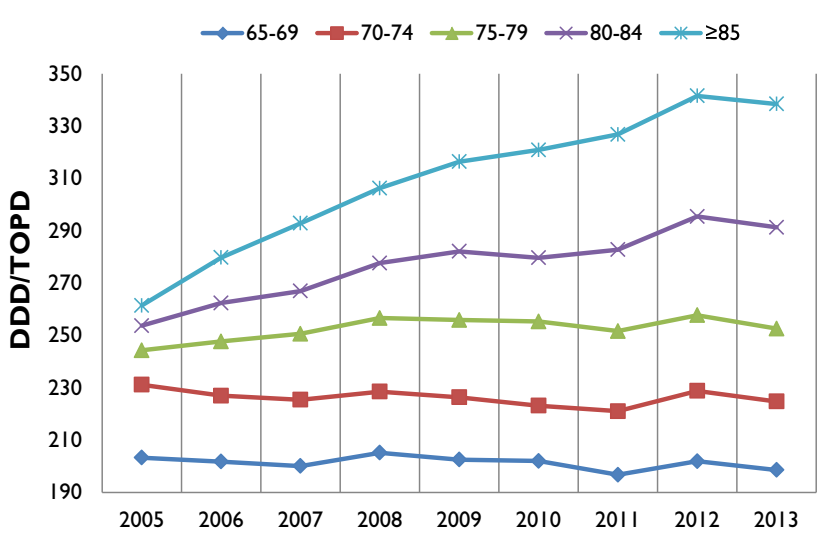

Fig. 2 DDD/TOPD utilization based on age groups from 2005 to 2013. $D D D$ defined daily dose, TOPD 1000 older people per day

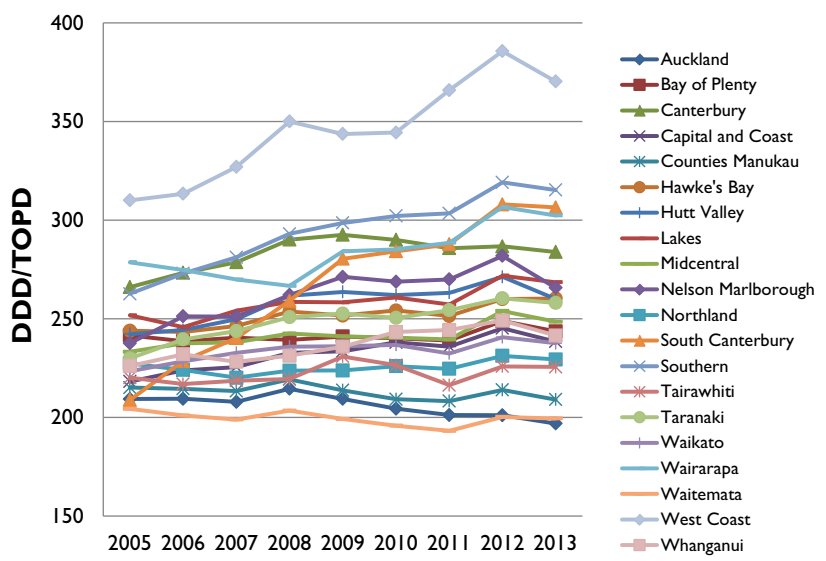

Fig. 3 DDD/TOPD utilization based on DHBs from 2005 to 2013. $D D D$ defined daily dose, DHBs District Health Boards, TOPD 1000 older people per day

309.37 DDD/TOPD (53.63\% higher than for the 65-69 age group).

As shown in Fig. 3, analgesic utilization was highest in the West Coast DHB, followed by the Southern DHB. 
Conversely, the Capital and Coast DHB had the lowest analgesic utilization. Overall, the differences in analgesic utilization across the DHBs persisted over the 9-year period. Only the South Canterbury DHB showed a steeper increase over the years when compared with other DHBs.

As shown in Fig. 1, females utilized more analgesic medicines compared with males. As for ethnicity, Middle Eastern, Latin American or African (MELAA), New Zealand European and Pacific groups had similar analgesic utilization, although, on average, New Zealand European individuals consumed the highest amount of analgesics (average DDD/TOPD 257.45). Analgesic utilization was lowest among the Asian ethnic group (average DDD/TOPD of 157.67), followed by the Māori group (average DDD/ TOPD of 232.81), as shown in Fig. 4.

\subsection{Utilization of Analgesic Medicines by Therapeutic Subgroups}

Anti-migraines had the lowest DDD utilization (average DDD/TOPD of 0.73) during the study period, followed by opioids (average DDD/TOPD of 51.37), as shown in Fig. 5. Overall, utilization of anti-migraines was low and relatively constant over the years. The utilization of

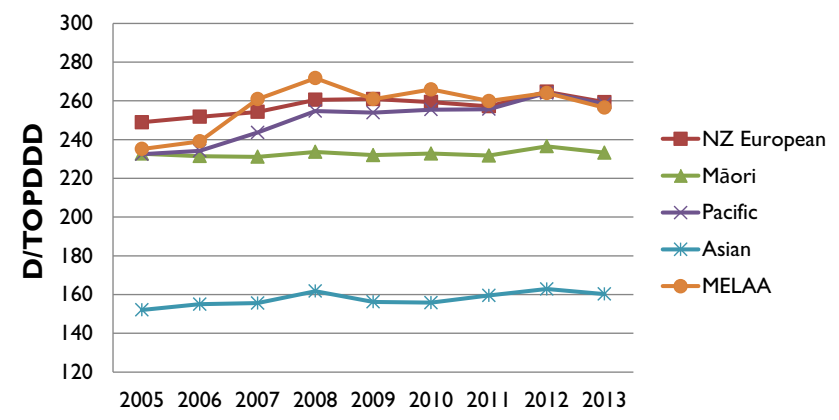

Fig. 4 DDD/TOPD utilization based on ethnicity from 2005 to 2013. $D D D$ defined daily dose, MELAA Middle Eastern, Latin American or African, NZ New Zealand, TOPD 1000 older people per day

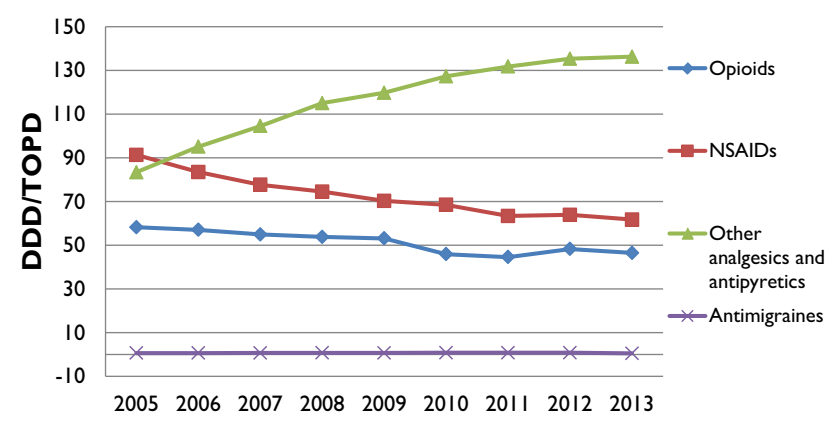

Fig. 5 DDD/TOPD utilization based therapeutic subgroups from 2005 to 2013. DDD defined daily dose, NSAIDs non-steroidal antiinflammatory drugs, TOPD 1000 older people per day analgesics and antipyretics increased over the 9-year period, and in contrast, utilization of opioids and NSAIDs declined. Analgesics and antipyretics had the highest DDD among the therapeutic subgroups, since 2006, with an average DDD/TOPD of 116.50 .

\subsection{Utilization of Analgesic Medicines by Pharmacological/Chemical Subgroups}

The highest increase in analgesic medicine utilization from 2005 to 2013 based on the pharmacologic/chemical subgroups were phenylpiperidine derivatives ( $\mathrm{N} 02 \mathrm{AB})$, mainly fentanyl, followed by natural opium derivatives (N02AA) (oxycodone). On the contrary, aspirin, from the salicylic acid and derivatives (N02BA) subgroup, had the highest decrease in utilization over the study period.

The most frequently prescribed analgesic over the 9-year period was paracetamol, from the anilides (NO2BE) subgroup, with an average DDD/TOPD of 132.49. Diclofenac, from the acetic acid derivatives and related substances (M01AB) subgroup, was ranked the second highest, followed by naproxen, from the propionic acid derivatives (M01AE) subgroup, with DDD/TOPDs of 29.06 and 17.85 , respectively. Mefenamic acid, from the fenamates (M01AG) subgroup, had the lowest average DDD/TOPD of 0.005 , and no change in the utilization rate was observed from 2005 to 2013. For anti-migraine preparations, sumatriptan had the highest decline in utilization, with an average DDD/TOPD of 0.59 .

\section{Discussion}

\subsection{Overall Trend}

Overall analgesic utilization in older people (65 years and above) increased by $5.44 \%$ from 2005 to 2013. A population-based study in France showed a high prevalence of pain $(72 \%)$ in the $65+$ community [3]. One in three participants (aged 75 years and above) in the study done by Hartikainen et al. [7] had daily interfering pain and one in ten suffered from daily resting pain. Because of the aging population in New Zealand (average $1.7 \%$ increase annually for the population aged 65-79 and $3.5 \%$ for those $80+$ ) [18], perhaps it is not surprising that analgesic utilization increased from 2005 to 2013, since the prevalence of pain is high in this age group [3, 4, 19]. Studies assessing the prevalence of pain in the community reported pain was usually under-treated among older people $[3,19]$. It is plausible that increased recognition, assessment and treatment of pain in older people may have contributed to increased utilization of analgesics over the study period. 
As mentioned in the Sect. 1, medication payment methods might result in changes of the analgesic utilization profiles. There are three ways that medications are paid in New Zealand: government funding (via PHARMAC), outof-pocket payment and coverage by insurance companies, which account for 82,11 and $5 \%$ of the total health expenditure in New Zealand, respectively [10]. PHARMAC's role has expanded over the years since its establishment in 1993. Apart from managing the medicines budget, PHARMAC also advocates the optimal use of medicines, making sure that best health outcomes are achieved within the allocated funding [11]. PHARMAC decides on medicine subsidies in different clinical settings [11], and therefore plays a significant role in influencing analgesic medicine utilization in New Zealand. The usage of certain types of analgesics will be prioritized on the basis of the criteria set by PHARMAC periodically, resulting in different analgesic utilization rates over the years.

\subsection{Utilization by Age, Sex, Ethnicity and DHB}

Older adults aged 85 years and above had the highest rate of analgesic medicine utilization, $53.63 \%$ higher than the 65-69 age group. This finding is consistent with chronic pain being common among older age groups [3, 4, 19]. The study conducted by Hartikainen et al. [7] showed that the utilization of analgesic medicines was highest among those older than 85 years. Similarly, another population-based study, conducted in Helsinki, Finland, over a 10-year period (1989-1999), showed that the utilization of analgesic medicines was higher in the age group of $85+$ compared with in those aged 75 years [19]. A study conducted by Blackwell et al. [13] assessing the number of analgesic medications refills showed that people older than $85+$ had the highest refill rate. In contrast, an investigation carried out in Italy showed that older adults aged $85+$ were least likely to receive analgesic medications. The investigators hypothesized that poor pain assessments and physical barriers such as payment methods, state regulations and restrictions to opioids may have contributed to low analgesic utilization [20]. Older people may not report pain unless being prompted; hence, using an appropriate pain assessment tool could be helpful in identifying people with undiagnosed and undertreated pain [21].

Our study finding of increased analgesic utilization by females was consistent with Blackwell et al.'s finding that females had higher analgesic refill rates compared with males and the results of two other studies, carried out in the USA and Manitoba [13, 22, 23]. A cross-sectional postal survey conducted in Scotland reported that females had a higher probability of using more non-prescription medicines, particularly analgesics, compared with males [24]. These differences may be attributed to the fact that females have a higher prevalence of pain associated with medical conditions [25] and a higher sensitivity to painful stimuli compared with males [26]. Gender roles and societal expectations may also lead to lesser reporting of pain among males compared with females [27], which can be translated to lower analgesic utilization.

New Zealand Europeans showed the highest utilization compared with other groups (average DDD/TOPD of 257.45). Our results are supported by a study described by Blackwell et al. [13], which showed that Europeans had a higher analgesic refill rate compared with other ethnic groups. A similar pattern was reported in a US populationbased study conducted by Paulose-Ram et al. [23], which showed a higher prevalence of non-prescription analgesics use among non-Hispanic whites; however, this study did not show any difference in the use of prescription analgesics between different ethnic groups. It is conceivable that these differences are brought about by discrepancies in the access to healthcare services [28]. The differences observed can also be explained by the fact that some ethnicities, especially Māori, are less likely to collect their prescriptions [29], leading to lower utilization of medications, including analgesics, compared with New Zealand Europeans.

It is important to note that analgesic utilization by MELAA ethnic groups has been relatively high over the years, even surpassing that of New Zealand European people between 2007 and 2011. MELAA is a term used in the New Zealand Census to describe a diverse group of people from different ethnicities originating from Middle Eastern, Latin American and African countries. While people categorized under this group make up only $1 \%$ of New Zealand's total population, this group consists of more than 55 different subgroups, each with unique health and socioeconomic needs [30]. It is therefore difficult to ascertain the real driver behind the high utilization reported. It has been reported that people in the MELAA ethnic group are less likely to seek treatment for mental health problems because of fear of stigmatization and lack of awareness [31]. A number of mental health conditions may manifest as physical pain [32], and since people from this group tend to under-report mental health problems, we can only speculate that this could be one of the many reasons for the substantial proportion of analgesics prescribed to them for somatization of psychiatric conditions.

Highest analgesic utilization was observed in the West Coast DHB. The West Coast's population was found to be older than the national average [33]. As our result suggested that the oldest people $(85+)$ consumed the most analgesics, this may be the reason for such high analgesic utilization in the West Coast DHB. More funding was available as well [33], because of their demographic profiles, thereby making prescribers more likely to prescribe 
analgesics. In contrast, we postulated that prescribers in the Waitemata DHB had the lowest tendency to prescribe analgesics. The population in the Waitemata DHB was found to be slightly younger than the national average [34]. On the basis of on our results, people in younger age require less analgesics; therefore, it is not surprising to see the Waitemata DHB had the lowest analgesic utilization. Variations in the utilization of analgesics across DHBs can also be explained by the way individuals refill their scripts, e.g. individuals enrolled in the Waitemata DHB may refill their prescriptions in Auckland. However, the true underlying causes for the trends in analgesic utilization across different DHBs were not known and further research is needed in order to ascertain such differences.

\subsection{Utilization by Therapeutic Subgroups}

It is noteworthy that utilization of NSAIDs in New Zealand decreased from 2005 to 2013, with a more prominent (more than $50 \%$ ) decline observed for tiaprofenic acid, ketoprofen, sulindac and tenoxicam. This is in contrast to the use of NSAIDs in other countries such as Costa Rica, where NSAID utilization increased by $48 \%$ from 2000 to 2005 [35]. The decrease in NSAID utilization is preferable in the clinical setting since the use of NSAIDs has been associated with adverse effects that can be detrimental in the elderly [36]. The drawbacks of NSAIDs (impacts on blood pressure, renal and cardiac functions, risk of gastrointestinal bleeding) have been largely discussed over the last few decades [19]. Older people are generally more prone to the adverse effects of NSAIDs because of their comorbidities (e.g. impaired renal function) [36], high drug exposures and drug sensitivity. Adverse events are further exacerbated by long-term use of NSAIDs, which leads to high drug exposure [37]. However, this option is still preferred in the UK because of its effectiveness against musculoskeletal complaints. Exploring the safest possible NSAIDs has been postulated as a possible solution to these concerns [37]. We could speculate that NSAID toxicities are generally well understood among the prescribers in New Zealand, which is evident from our results showing a decreased utilization of NSAIDs among older people. Best Practice Advocacy Centre New Zealand $\left(\mathrm{Bpac}^{\mathrm{nz}}\right)$ has highlighted the risks associated with NSAIDs, and cautions are warranted in all age groups even for short-term treatment [38]. $\mathrm{Bpac}^{\mathrm{nz}}$ also recommends the prophylactic use of gastro-protectants in those aged over 45 if prescribed longterm NSAIDs [38]. The decline in NSAID utilization may suggest that $\mathrm{Bpac}^{\mathrm{nz}}$ clinical guidelines are routinely followed by the prescribers.

The overall utilization of opioids in New Zealand declined from 2005 to 2013. This is in contrast to the findings from other regions of the world, e.g. Bosnia, Herzegovina and the USA, which showed an increasing trend over the years $[39,40]$. A study carried out in the USA analyzing opiate use showed an overall $309 \%$ increase from 1996 to 2002 , with a $170 \%$ rise in the market basket use [40]. The use of opioids in older people has been shown to be associated with cognitive impairment and falls [7]. A review on medication-related falls in the elderly conducted by Huang et al. [12] showed that women taking opioids were at increased risk of developing non-spine fractures and hip fractures [odds ratio (OR) of 1.40 and 1.65 , respectively] as well as recurrent falls (OR of 2.89). The study conducted by Miller et al. [41] found that older people with arthritis who initiated therapy with opioids were more likely to experience a fracture than those who initiated NSAIDs, and short-acting opioids were associated with a greater risk of fracture than long-acting opioids. In contrast to these findings, two meta-analyses, conducted by Woolcott et al. [42] and Leipzig et al. [43], respectively, found that opioid analgesics, compared with other classes of medications, e.g. antidepressants and antipsychotics (OR of 0.96 and 0.97 , respectively) had a lower risk associated with falls.

The study conducted by Blackwell et al. [13] suggested morphine and fentanyl as safe opioids for treating severe pain in the elderly because of a lower risk of injuries associated with their usage. At present, opioids are considered the cornerstone for chronic moderate to severe pain management in older people when paracetamol alone is not sufficient [1]. Close monitoring of opioids' adverse drug reactions as well as their effectiveness would be beneficial in the clinical setting when initiating opioid treatments in the elderly to ensure any harms associated with their use are minimized.

Access to opioid analgesics may influence analgesic utilization across countries with different healthcare systems. In New Zealand, laws and regulations play an important role in shaping analgesic utilization, particularly for opioid analgesics. Weak opioids such as codeine are available OTC when in combination with other active ingredients. Examples include Panadeine (paracetamol and codeine) and Nurofen Plus (ibuprofen and codeine). Codeine itself and all other opioid analgesics are classified as prescription-only medicines, where strict regulations are enforced [9]. In order to prescribe Class A and Class B controlled drugs [44] such as codeine, health professionals are required to fill in triplicate controlled drugs prescription forms (H572), and pharmacists must make sure all the legal requirements are fulfilled before dispensing the controlled medicine [45].

Anti-migraine preparation utilization among old people remained consistently low between 2005 and 2013. The average DDD/TOPD for sumatriptan in New Zealand was 0.59. This is consistent with the findings from other countries, such as Italy, which showed that utilization of 
triptans were low (total DDD/TOPD of 0.78) among people with migraines [46].

\subsection{Utilization of Analgesic Medicines by Pharmacological/Chemical Subgroups}

Fentanyl and oxycodone had the highest increase in their overall utilization over the 9 -year period. The rise was consistent with the studies done in other countries, namely the USA, Spain and Israel [1, 6, 8]. The researchers in the USA commented that prescription drug abuse had become prevalent with an increase in the prescription of oxycodone and hydrocodone [6]. The study in Spain found that fentanyl utilization was the major contributing factor for the 14-fold increase of Spain's overall opioid consumption, while another study carried out in Israel found that fentanyl utilization increased fourfold from 2000 to 2008 [1, 8]. The huge increase in New Zealand's fentanyl and oxycodone utilization warrants further research to investigate the true underlying contributing factors for the rise, as well as its impact on pain control and/or abuse rates.

High-dose aspirin is less commonly used as an analgesic or anti-inflammatory agent since by comparison NSAIDs such as ibuprofen, naproxen and diclofenac are proven to have better safety and efficacy profiles [47]. Aspirin has a more profound role as an antiplatelet agent in reducing cardiovascular risks [48]. This may be the reason for the highest decrease in aspirin utilization as an analgesic over the study period.

In terms of individual analgesic utilization, paracetamol had the highest DDD/TOPD, followed by diclofenac and naproxen, with mefenamic acid as the lowest. Paracetamol is well recognized as a safe and effective analgesic for long-term use [21], and it is generally well tolerated across all age groups [49], which may be the reason for it being the most widely used analgesic among older people. Diclofenac and naproxen are two analgesics with a lower incidence of adverse effects, and are preferred over other NSAIDs [50]. Diclofenac is available in various dosage forms (injection, suppository, dispersible tablet, entericcoated tablet, modified release tablet), as compared with naproxen (immediate release tablet, modified release tablet, enteric-coated tablet). A more diverse range of delivery methods provides a greater choice for both the patients and the prescribers, which may lead to higher utilization and might be the reason for diclofenac having the second highest DDD/TOPD. Mefenamic acid has minor anti-inflammatory properties, and it is mainly indicated for period pain, which is an unlikely health complaint among older people. Adverse effects such as diarrhoea and haemolytic anaemia were reported, which could be the reasons for its low utilization [50].

\subsection{Study Strengths and Limitations}

In New Zealand, policy makers have a major stake in the funding of analgesic prescriptions by evaluating clinical safety as well as the cost. Some changes in the utilization were largely driven by the funding decisions led by PHARMAC [11] and some were due to withdrawal of products from the market.

A limitation of our study is that the data contain no information regarding patient compliance to the therapy. The database only recorded all the dispensed prescription medications, without taking into consideration whether the patients actually consumed the dispensed medications. Moreover, OTC products purchased by individual patients were not included. Prescribing restrictions for opioid analgesics increases the reliability of opioid utilization, but this may not apply for NSAIDs. It is possible to speculate that the low utilization of NSAIDs may be due to exclusion of the OTC consumption of NSAIDs. Examples of OTC NSAIDs available in New Zealand include diclofenac, ibuprofen, mefenamic acid, naproxen and aspirin. The study also did not include analgesics that are not available or not funded in New Zealand; an important example are the selective inhibitors of cyclo-oxygenase-2 (coxibs), e.g. celecoxib. While coxibs are available in New Zealand as prescription medicines, they are not funded by PHARMAC [38]; therefore, it was difficult to determine the real extent of their utilization in New Zealand. This is a significant limitation, as the use of coxibs is associated with a higher risk of adverse cardiovascular events, especially in older people [51]. Another significant limitation is the lack of diagnostic data to corroborate analgesic utilization in this population.

\section{Conclusions}

This population level utilization study showed a $5.44 \%$ increase in the utilization of analgesic medicines in older people in New Zealand from 2005 to 2013. Analgesics under the N02B therapeutic subgroup (other analgesics and antipyretics), represented by paracetamol, are a major contributor to the increase in utilization over the years. Important findings were that (1) females utilized more analgesics compared with males; (2) NSAID utilization decreased over the years; and (3) the highest utilization was reported in the $85+$ group. Further studies need to be carried out to investigate the drivers behind the rise in analgesic utilization over the years, as well as its impact on the health and wellbeing of older people in New Zealand.

Acknowledgments The authors thank Analytical Services, Ministry of Health of New Zealand, for supplying the dispensing data extracted from the Pharms database. 
Conflict of interest P.S. Nishtala, S. Oh, D. Kim, N. Chun, S.F. Kamis and K.-C. Kiu declare that they have no conflicts of interest to declare.

Open Access This article is distributed under the terms of the Creative Commons Attribution-NonCommercial 4.0 International License (http://creativecommons.org/licenses/by-nc/4.0/), which permits any noncommercial use, distribution, and reproduction in any medium, provided you give appropriate credit to the original author(s) and the source, provide a link to the Creative Commons license, and indicate if changes were made.

\section{References}

1. Garcia del Pozo J, Carvajal A, Viloria J, Velasco A, Garcia del Pozo V. Trends in the consumption of opioid analgesics in Spain. Higher increases as fentanyl replaces morphine. Eur J Clin Pharmacol. 2008;64(4):411-5.

2. Ponizovsky AM, Pchelintsev MV, Marom E, Zvartau EE. Differences in the consumption rates and regulatory barriers to the accessibility of strong opioid analgesics in Israel and St. Petersburg. Eur J Clin Pharmacol. 2012;68:89-95.

3. Brochet B, Michel P, Barberger-Gateau P, Dartigues J-F. Population-based study of pain in elderly people: a descriptive survey. Age Ageing. 1998;27(3):279.

4. Crook J, Rideout E, Browne G. The prevalence of pain complaints in a general population. Pain. 1984;18(3):299-314.

5. Cicero TJ, Inciardi JA, Surratt H. Trends in the use and abuse of branded and generic extended release oxycodone and fentanyl products in the United States. Drug Alcohol Depend. 2007;91(2):115-20.

6. Cicero TJ, Inciardi JA, Muñoz A. Trends in abuse of OxyContin ${ }^{\circledR}$ and other opioid analgesics in the United States: 2002-2004. J Pain. 2005;6(10):662-72.

7. Hartikainen SA, Mäntyselkä PT, Louhivuori-Laako KA, Sulkava RO. Balancing pain and analgesic treatment in the home-dwelling elderly. Ann Pharmacother. 2005;39(1):11.

8. Ponizovsky AM, Marom E, Zeldin A, Cherny NI. Trends in opioid analgesics consumption, Israel, 2000-2008. Eur J Clin Pharmacol. 2011;67:165-8.

9. New Zealand Ministry of Health. Frequently asked questions: controlled drugs prescribing. 2011 [cited 20155 Mar]. Available from: https://www.health.govt.nz/system/files/documents/pages/ controlled-drugs-prescribing-v1.pdf.

10. Gauld R. The New Zealand Health Care System, 2013. In: Thomson S, Osborn R, Squires D, Jun M, editors. International Profiles of Health Care Systems, 2013: Australia, Canada, Denmark, England, France, Germany, Italy, Japan, the Netherlands, New Zealand, Norway, Sweden, Switzerland, and the United States: The Commonwealth Fund; 2013. p. 94-102.

11. Cumming J, Mays N, Daubé J. How New Zealand has contained expenditure on drugs. BMJ. 2010;340:c2441.

12. Huang AR, Mallet L, Rochefort CM, Eguale T, Buckeridge DL, Tamblyn R. Medication-related falls in the elderly. Drugs Aging. 2012;29(5):359-76.

13. Blackwell SA, Baugh DK, Montgomery MA, Ciborowski GM, Levy JM. National study of injury-increasing analgesics in the elderly. Int J Pharm Healthc Mark. 2011;5(1):25-42.

14. Hajjar ER, Cafiero AC, Hanlon JT. Polypharmacy in elderly patients. Am J Geriatr Pharmacother. 2007;5(4):345-51.

15. Andersson HI, Ejlertsson G, Leden I, Rosenberg C. Chronic pain in a geographically defined general population: studies of differences in age, gender, social class, and pain localization. Clin J Pain. 1993;9(3):174-82.

16. Bates MS, Edwards WT. Ethnic variations in the chronic pain experience. Ethn Dis. 1992;2(1):63-83.

17. Statistics NZ. Census QuickStats about national highlights. New Zealand: Statistics New Zealand; 2013 [cited 201430 Mar]. Available from: http://www.stats.govt.nz/Census/2013.

18. Statistics New Zealand Tatauranga Aotearoa. National population estimates: June 2009 quarter. [cited 201415 Aug]. Available from: http://www.stats.govt.nz/browse_for_stats/population/ estimates_and_projections/NationalPopulationEstimates_HOTP Jun09qtr/Commentary.

19. Pitkala KH, Strandberg TE, Tilvis RS. Management of nonmalignant pain in home-dwelling older people: a population-based survey. J Am Geriatr Soc. 2002;50(11):1861-5.

20. Landi F, Onder G, Cesari M, Gambassi G, Steel K, Russo A, et al. Pain management in frail, community-living elderly patients. Arch Intern Med. 2001;161(22):2721.

21. Best Practice Advocacy Centre New Zealand. Recognition and treatment of pain in elderly people. [cited 201415 Aug]. Available from: http://www.bpac.org.nz/BPJ/2008/February/pain.aspx.

22. Sadowski CA, Carrie AG, Grymonpre RE, Metge CJ, St. John P. Access and Intensity of use of prescription analgesics among older Manitobans. Can J Clin Pharmacol. 2009;16(2):e322-30.

23. Paulose-Ram R, Hirsch R, Dillon C, Losonczy K, Cooper M, Ostchega Y. Prescription and non-prescription analgesic use among the US adult population: results from the third National Health and Nutrition Examination Survey (NHANES III). Pharmacoepidemiol Drug Saf. 2003;12(4):315-26.

24. Porteous T, Bond C, Hannaford P, Sinclair H. How and why are non-prescription analgesics used in Scotland? Fam Pract. 2005;22:78-85.

25. Isacson D, Bingefors K. Epidemiology of analgesic use: a gender perspective. Eur J Anaesthesiol. 2002;19(Supplement 26):5-15.

26. Riley Iii JL, Robinson ME, Wise EA, Myers CD, Fillingim RB. Sex differences in the perception of noxious experimental stimuli: a meta-analysis. Pain. 1998;74(2):181-7.

27. Robinson ME, Riley JL, Myers CD, Papas RK, Wise EA, Waxenberg LB, et al. Gender role expectations of pain: relationship to sex differences in pain. J Pain. 2001;2(5):251-7.

28. Malcolm L. Inequities in access to and utilisation of primary medical care services for Maori and low income New Zealanders. N Z Med J. 1996;109(1030):356-8.

29. New Zealand Ministry of Health. Tatau Kahukura: Māori Health Chart Book 2010. 2010 [cited 201427 Sept]. Available from: http://www.health.govt.nz/publication/tatau-kahukura-maorihealth-chart-book-2010-2nd-edition.

30. Statistics New Zealand Tatauranga Aotearoa. Census ethnic group profiles. 2013 [cited 20155 Mar]. Available from: http:// www.stats.govt.nz/Census/2013-census/profile-and-summaryreports/ethnic-profiles.aspx.

31. Perumal L. Health needs assessment of Middle Eastern, Latin American and African people living in the Auckland region. Auckland: Auckland District Health Board; 2010.

32. Gureje O, Simon GE, Ustun TB, Goldberg DP. Somatization in cross-cultural perspective: a World Health Organization study in primary care. Am J Psychiatry. 1999;154(7):989-95.

33. New Zealand Ministry of Health. Population of West Coast DHB. 2014 [cited $201420 \mathrm{Sept}$. Available from: http://www.health. govt.nz/new-zealand-health-system/my-dhb/west-coast-dhb/ population-west-coast-dhb.

34. New Zealand Ministry of Health. Population of Waitemata DHB. 2014 [cited 201420 Sept]. Available from: http://www.health. govt.nz/new-zealand-health-system/my-dhb/waitemata-dhb/ population-waitemata-dhb. 
35. Morera Salas M, Aparicio Llanos A, Xirinachs Salazar Y, Barber Perez P. Consumption of nonsteroidal anti-inflammatory agents in primary care in Costa Rica: changing patterns and geographical variability [Spanish]. Gaceta Sanitaria. 2007;21(6):458-64.

36. Waitemate District Health Board. Triple whammy - safe prescribing — a dangerous trio. 2014 [cited 201411 Sept]. Available from: http://www.saferx.co.nz/full/triplewhammy.pdf.

37. Hawkey CJ, Cullen DJE, Pearson G, Holmes S, Doherty M, Wilson JV, et al. Pharmacoepidemiology of non-steroidal antiinflammatory drug use in Nottingham general practices. Aliment Pharmacol Ther. 2000;14(2):177-85.

38. Best Practice Advocacy Centre New Zealand. Non-steroidal antiinflammatory drugs (NSAIDs): making safer treatment choices. [cited 201416 Aug]. Available from: http://www.bpac.org.nz/ bpj/2013/october/nsaids.aspx.

39. Marković-Peković V, Stoisavljević-Šatara S, Škrbić R. Outpatient utilisation of drugs acting on nervous system: a study from the Republic of Srpska, Bosnia \& Herzegovina. Eur J Clin Pharmacol. 2010;66:177-86.

40. Zerzan JT, Morden NE, Soumerai S, Ross-Degnan D, Roughead $\mathrm{E}$, Zhang $\mathrm{F}$, et al. Trends and geographic variation of opiate medication use in state Medicaid Fee-For-Service programs, 1996 to 2002. Med Care. 2006;44(11):1005-10.

41. Miller M, Stürmer T, Azrael D, Levin R, Solomon DH. Opioid analgesics and the risk of fractures in older adults with arthritis. J Am Geriatr Soc. 2011;59(3):430-8.

42. Woolcott JC, Richardson KJ, Wiens MO, Patel B, Marin J, Khan $\mathrm{KM}$, et al. Meta-analysis of the impact of 9 medication classes on falls in elderly persons. Arch Intern Med. 2009;169(21):1952-60.
43. Leipzig RM, Cumming RG, Tinetti ME. Drugs and falls in older people: a systematic review and meta-analysis: II. Cardiac and analgesic drugs. J Am Geriatr Soc. 1999;47(1):40-50.

44. Legislation NZ. Misuse of Drugs Act 1975. 2015 [cited 201522 Apr]. Available from: http://www.legislation.govt.nz/act/public/ 1975/0116/latest/DLM436576.html.

45. New Zealand Ministry of Health. Funding. 2014 [cited 20151 Mar]. Available from: http://www.health.govt.nz/new-zealandhealth-system/overview-health-system/funding.

46. Panconesi A, Pavone E, Vacca F, Vaiani M, Banfi R. Triptans in the Italian population: a drug utilization study and a literature review. J Headache Pain. 2008;9(2):71-6.

47. Garcia Rodriguez LA, Hernandez-Diaz S, de Abajo FJ. Association between aspirin and upper gastrointestinal complications: systematic review of epidemiologic studies. Br J Clin Pharmacol. 2001;52(5):563-71.

48. Ittaman SV, VanWormer JJ, Rezkalla SH. The role of aspirin in the prevention of cardiovascular disease. Clin Med Res. 2014;12(3-4):147-54.

49. Forte JS. Paracetamol: safety versus toxicity. Chronic ill. 2002;6:12-6.

50. New Zealand Formulary. Non-steroidal anti-inflammatory drugs. 2014 [cited 201409 Sept]. Available from: http://www.nzf.org. nz/nzf_5476_all.html.

51. Farkouh ME, Greenberg BP. An evidence-based review of the cardiovascular risks of nonsteroidal anti-inflammatory drugs. Am J Cardiol. 2009;103(9):1227-37. 